\title{
Computer simulation of
}

\section{polymethylmethacrylate bone cement flow through femoral canal and cancellous bone}

\author{
S. Srimongkol* \\ B. Wiwatanapataphee* \\ Y. H. $\mathrm{Wu}^{\dagger}$
}

(Received 28 October 2005; revised 18 August 2006)

\begin{abstract}
Polymethylmethacrylate bone cements have been widely used in orthopaedic surgery for the fixation of artificial joints such as hip replacement. Understanding the flow of cement is crucial to the success of the process, as the cement flow determines the final shape of the filling and consequently the strength and durability of the structure. A mathematical model based on the finite element method is developed to simulate the flow of cement through femoral canal and cancellous bone. The cement is assumed as a power law non-Newtonian fluid
\end{abstract}

${ }^{*}$ Dept. of Mathematics, Faculty of Science, Mahidol University, Bangkok, THAILAND. mailto:scbww@mahidol.ac.th

$\dagger$ Dept. of Mathematics and Statistics, Curtin Univ. of Tech., Western Australia. mailto:yhwu@maths . curtin. edu . au

See http://anziamj.austms.org.au/V47EMAC2005/Srimongkol for this article, (C) Austral. Mathematical Soc. 2006. Published October 23, 2006. ISSN 1446-8735 
and its flow in femoral canal is governed by the non-linear NavierStokes equations. The cancellous bone is modelled as a porous media and the Brinkman equations are used to describe the flow of cement through the bone. Based on the model developed, various numerical experiments were carried out to investigate the influence of cement property and surgical conditions on flow pattern, pressure distribution and shear rate.

\section{Contents}

1 Introduction

C405

2 Mathematical model

C408

3 Numerical methods

C411

4 Numerical results and discussion

C412

5 Conclusions

C415

References

C415

\section{Introduction}

Bones produce blood cells and store minerals such as calcium and phosphate. They have to be strong enough to support human weight and protect human internal organs. There are two major types of bones, flat bone and long bone. Flat bone consists of thin layers of bone and thin layers of marrow. Rib is an example of flat bone. Long bone which is long, hard and dense is made of connective tissue. It contains yellow marrow and red marrow which produce blood cells. Femur is an example of long bone. 
Bone may be painful from many causes such as fracture, osteoarthritis and rheumatoid arthritis. In the case that the bone was damaged at the hip joint due to the severe arthritis in the hip, the ability for the patients to do their normal activities is limited. If fractures occur in the elderly of the neck of the femur or tumors occur in the hip joint, hip replacement known as arthroplasty is needed.

In the total hip replacement process, surgeons replace all or a part of the hip joint with a prosthesis. The total hip prosthesis consists of three parts. The first part is a cup which is used to replace a hip socket. The cup may be made of plastic, ceramic or metal. The second part is a metal or ceramic ball which is used to replace the fracture head of the femur. The last part is a metal stem which is attached to the shaft of the bone to increase the stability of the prosthesis. If the surgeon does a hemi-arthroplasty, the bone is replaced with a prosthetic device only in the head of the femur. The surgeon starts performing the total hip replacement by using general or spinal anesthesia. Then the orthopedic surgeon makes an incision over the buttocks to expose the hip joint. After that the head of the femur is cut out and removed. The hip socket is cleaned out. A reamer is then used to remove all of the remaining cartilage and arthritic bone. The new socket is implanted after the metal stem is inserted into the femur. The artificial components are fixed sometimes with a special cement. Finally, the muscles and tendons are replaced against the bones. In the case that the orthopedic surgeon uses cement in the operation, the cement is firstly injected in the hip canal and the prosthesis is then implanted.

Although the success rate in hip replacement is very high, implant failure or implant loosening still happens [12]. Generally, the average life span of an implant is about 15 years; eventually it wears out. This causes more problems to younger patients who live longer and hence place more demands on the implanted joint. If the joint wears out, a revision replacement may be performed. Blood clots, dislocation and infection are also problems occurring in hip replacement. Turner et al. [15] pointed out that the failure or loosening 
of implants is often associated with stress shielding which is caused by load sharing between bone and implant. Some clinical studies show that implant loosening usually starts on the bone-cement interface and the stem-cement interface [13].

The cement Polymethylmethacrylate (PMMA) has been widely used in orthopedic surgery over the last four decades [14]. It has been used for the fixation of fractures in osteoporotic bone and the fixation of artificial joints such as hip replacement. Since PMMA cement has no adhesive property [14], a strong and durable cement-bone interface is important [8]. The loosening femoral components in the cemented hip replacement may cause revision arthroplasty. The interlock between the cement-bone interface is generated when the cement is injected in doughty state and polymerizes in situ. Many problems in a laboratory still occur, such as errors from the evaluation of the cement pressurization equipment, cementation techniques, and the communication of appropriate techniques. Cement pressurization is required to ensure that the cement flows through the cancellous bone.

In order to increase the life span of an implant, a number of in vivo and in vitro experiments have been conducted $[1,4,5]$. McCaskie et al. [10] tried to improve interlock between cement and bone around the femoral stem by using high pressure and reducing viscosity of cements. Funk and Litsky [7] performed shear tests of the bone-cement interface in vitro using PMMA and polybutyl methacrylate. Lennon and Prendergast [9] investigated the residual stress due to shrinkage of PMMA bone cement after polymerization which is an important factor capable of initiating cracks in the mantle of cemented hip replacements. They concluded that cracks were induced by residual stress around pores or stress raisers.

Darcy's law was used to model the penetration of the PMMA bone cement into the cancellous bone which is a porous media $[2,3,6]$. Beaudoin et al. [3] developed a mathematical model using Darcy's law and based on the finite element method to simulate the acrylic bone cement flow through the cancellous bone. Their model predicts a cancellous bone permeability 
between $5.6 \times 10^{-9}$ to $8.3 \times 10^{-9} \mathrm{~m}^{2}$ for linear flow. Recently, Frei et al. [6] used the finite element method to simulate the cement flow during impaction allografting. Based on Darcy's law, the cement flux in tubes is

$$
Q=\frac{r^{2} \Delta p}{8 \eta L} A,
$$

where $Q$ is the volumetric flow rate $\left[\mathrm{m}^{3} \mathrm{~s}^{-1}\right], \Delta p$ is the applied pressure $[\mathrm{Pa}]$, $L$ is the length $[\mathrm{m}], A$ is the averaged cross sectional area $\left[\mathrm{m}^{2}\right]$ of the femoral canal, $\eta$ is the dynamic viscosity $[\mathrm{Pa}]$ and $r$ is the average radius $[\mathrm{m}]$ of the canal.

Although a large number of studies on cement flow have been conducted [2, $3,6,10]$ there have only been a few numerical studies on the cement flow in both the femoral canal and the spongy bone. The aim of this work is to propose a two dimensional finite element numerical method to fully couple the cement flow in the femoral canal and the spongy bone. The effect of the pressure used at the inlet where the cement is injected is investigated.

\section{Mathematical model}

Bones contain blood vessels, nerve cells and living bone cells known as osteocyte. The outer hard part contains non-living material, calcium and phosphorous. The surface of the bone is covered with a thin membrane, namely periosteum. The center of the long bone, such as a femur, is occupied by red bone marrow and yellow bone marrow. The red bone marrow is a soft tissue containing stem cells which produce blood cells, whereas the yellow bone marrow stores fat. The hard part of the bone is either spongy or compact: spongy is light weight and is made up of connective tissues with large space between them; compact bone is dense and forms the outer layer of the bones. The structure of the femur is shown in Figure 1. 


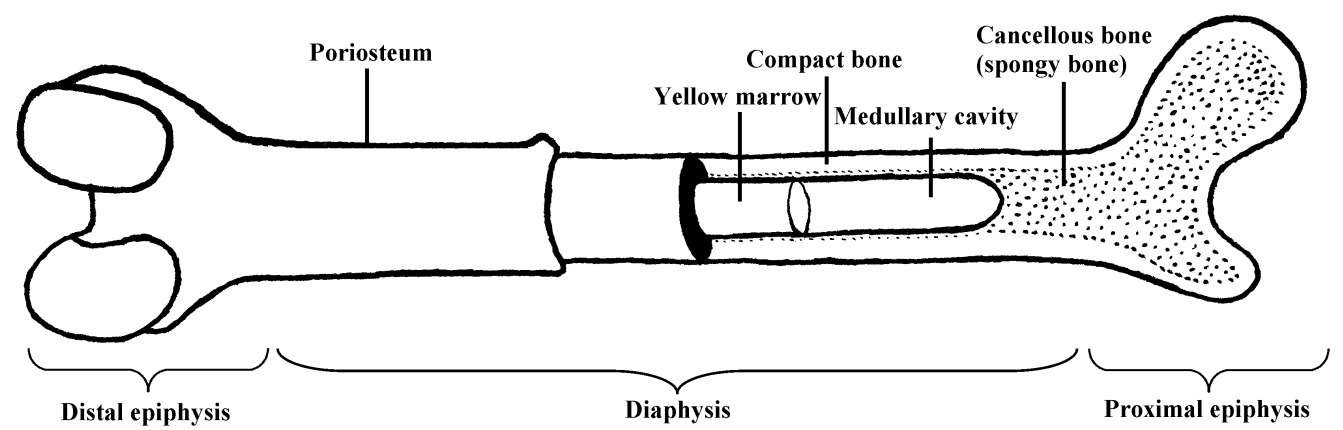

Figure 1: Structure of the long bone

We model the cement flow in two different parts of the bone: femoral canal (or marrow canal) and spongy bone. In the femoral canal, $\Omega_{1}$, we model the cement as a non-Newtonian fluid and its flow is governed by the following equations

$$
\begin{aligned}
& \nabla \cdot \mathbf{u}=0, \\
& \rho \frac{\partial \mathbf{u}}{\partial t}-\nabla \cdot \tau_{m}+\rho(\mathbf{u} \cdot \nabla) \mathbf{u}+\nabla p_{m}=\mathbf{F},
\end{aligned}
$$

where $\rho$ is the density of the fluid, $\mathbf{u}$ is the velocity of fluid in the marrow canal, $t$ is time, $p_{m}$ is the pressure in the marrow canal, $\mathbf{F}$ represents the gravitational force, $\tau_{m}$ is the shear stress tensor depending on the rate of the deformation tensor $\mathbf{D}$ by the relation $\tau_{m}=2 \eta(\dot{\gamma}) \mathbf{D}$, where $\mathbf{D}=\frac{1}{2}(\nabla \mathbf{u}+$ $\left.(\nabla \mathbf{u})^{T}\right), \eta$ is the viscosity of the fluid and $\dot{\gamma}$ is the shear rate. We use the power law model to determine the viscosity of the PMMA bone cement:

$$
\eta=m \dot{\gamma}^{n-1},
$$

where $m$ and $n$ are material parameters and $\dot{\gamma}=\sqrt{2 \operatorname{tr}\left(\mathbf{D}^{2}\right)}$.

In the cancellous bone or spongy bone, $\Omega_{2}$, we use the Brinkman equations 
to model the flow in the porous media:

$$
\begin{aligned}
& \nabla \cdot \mathbf{v}=0 \\
& \rho \frac{\partial \mathbf{v}}{\partial t}-\nabla \cdot \tau_{c}+\frac{\eta}{\kappa} \mathbf{v}+\nabla p_{c}=\mathbf{F},
\end{aligned}
$$

where $\mathbf{v}$ is the velocity of fluid in the cancellous bone, $p_{c}$ is the pressure in the cancellous bone, $\kappa$ is the permeability of the cancellous bone.

The field equations (2)-(3) for the flow of cement in $\Omega_{1}$ are subjected to the boundary conditions

$$
\begin{array}{lll}
p_{m}=p_{0}, & \tau_{m}=0 & \text { on } \Gamma_{1}, \\
p_{m}=p_{c}, & \tau_{m}=0 & \text { on } \Gamma_{2} .
\end{array}
$$

The continuity equation and the Brinkman equations for the flow in the domain $\Omega_{2}$ are subject to the boundary conditions

$$
\begin{aligned}
& \mathbf{v}=\mathbf{u} \quad \text { on } \Gamma_{2}, \\
& \mathbf{t} \cdot \mathbf{v}=0, \quad p_{c}=0, \quad \tau_{c}=0 \quad \text { on } \Gamma_{3} .
\end{aligned}
$$

We use $p_{0}$ as the initial pressure of the cement. Boundary conditions (8) and (9) are used on the interface between the marrow canal and the cancellous bone to smooth the flow on the interface. We use the neutral boundary condition (10) as the boundary condition for the cancellous bone which is a compact bone. We assume that there is no pressure on the interface between the cancellous bone and the compact bone. In brief, the underlying boundary value problem is:

Find $\mathbf{u}, \mathbf{v}, p_{m}$ and $p_{c}$ such that equations (2) and (3) are satisfied in domain $\Omega_{1}$ and equations (5) and (6) are satisfied in the domain $\Omega_{2}$ and all boundary conditions (7), (8), (9) and (10) are satisfied. 


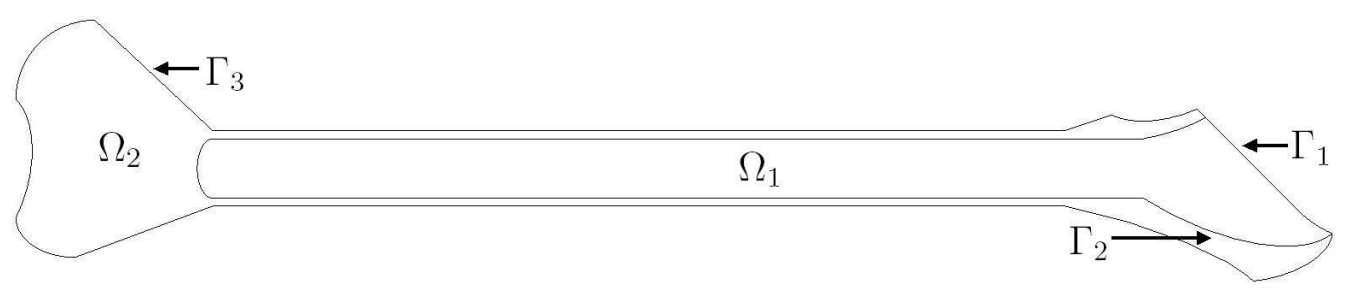

FIgURE 2: The 2-D femur bone consisting of the marrow canal $\left(\Omega_{1}\right)$ and the cancellous bone $\left(\Omega_{2}\right)$.

\section{Numerical methods}

To develop the variational statement for the problem, we consider the following alternative problem.

Find $\mathbf{u}, \mathbf{v}, p_{m}$ and $p_{c} \in H^{1}(\Omega)$ such that for all $\hat{\mathbf{u}}, \hat{\mathbf{v}}, \hat{p}_{m}$, and $\hat{p}_{c} \in H_{0}^{1}(\Omega)$, all boundary conditions (7), (8), (9) and (10) are satisfied and

$$
\begin{aligned}
& \int_{\Omega_{1}} \hat{p}_{m}(\nabla \cdot \mathbf{u}) d \Omega=0 \\
& \int_{\Omega_{1}} \hat{\mathbf{u}}^{\mathrm{T}}\left(\rho \frac{\partial \mathbf{u}}{\partial t}-\nabla \cdot \tau_{m}+\rho(\mathbf{u} \cdot \nabla) \mathbf{u}+\nabla p_{m}-\mathbf{F}\right) d \Omega=0 \\
& \int_{\Omega_{2}} \hat{p}_{c}(\nabla \cdot \mathbf{v}) d \Omega=0 \\
& \int_{\Omega_{2}} \hat{\mathbf{v}}^{\mathrm{T}}\left(\rho \frac{\partial \mathbf{v}}{\partial t}-\nabla \cdot \tau_{c}+\frac{\eta}{\kappa} \mathbf{v}+\nabla p_{c}-\mathbf{F}\right) d \Omega=0
\end{aligned}
$$

where $H^{1}(\Omega)$ is the Sobolev space $W^{1,2}(\Omega)$ with norm $\|\cdot\|_{1,2, \Omega}$, and $H_{0}^{1}(\Omega)=$ $\left\{v \in H^{1}(\Omega) \mid v=0\right.$ on Dirichlet type boundary $\}$.

Standard procedures for the development of a Galerkin finite element 


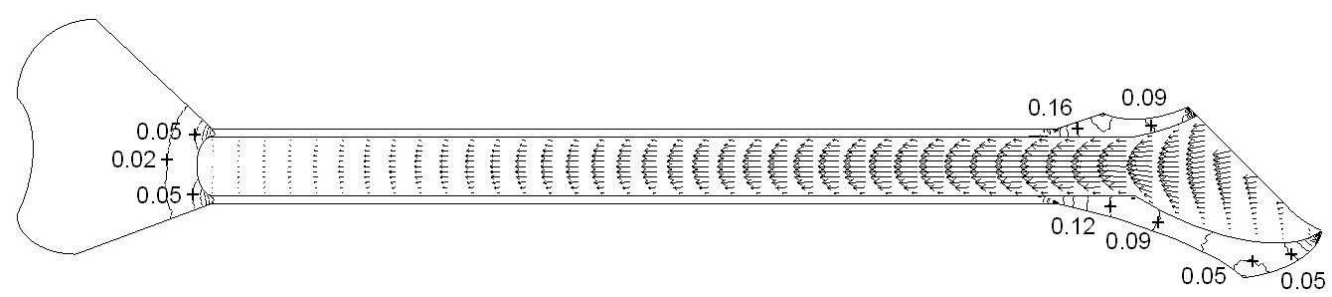

Figure 3: Velocity $(\mathrm{cm} / \mathrm{s})$ in the femoral canal and the cancellous bone.

formulation lead to a system of the form

$$
\begin{aligned}
C_{m}^{\mathrm{T}} \mathbf{U} & =0, \\
M_{m} \dot{\mathbf{U}}_{m}+D_{u} \mathbf{U}+A_{m}(\mathbf{U}) \mathbf{U}-C_{m} P_{m}+Q_{m} P_{c} & =R+F, \\
C_{c}^{\mathrm{T}} \mathbf{V} & =0, \\
M_{c} \dot{\mathbf{V}}+D_{v} \mathbf{V}+B_{c} \mathbf{V}-C_{c} P_{c}+Q_{c} P_{c} & =F .
\end{aligned}
$$

This set of equations are solved by the backward Euler time stepping scheme integrated with an iterative procedure dealing with the nonlinearity in the system.

\section{Numerical results and discussion}

An example for a typical femur bone is given here to study the flow of the PMMA cement through the cancellous bone. The example bone is the femur bone of height of $33.4 \mathrm{~cm}$. The density of the PMMA bone cement used here is $1.1 \mathrm{~g} / \mathrm{cm}^{3}$ [14], the permeability of the cancellous bone is $7.84 \times 10^{-5} \mathrm{~cm}^{2}$ [3], the power law parameters $m=269$ and $n=0.67$ [6], the initial pressure $p_{0}=$ $3.5 \times 10^{5}$ dyne $/ \mathrm{cm}^{2}[3,11]$ and the average viscosity used in the cancellous bone is 586.5 poise $(\mathrm{P})$ [3]. The finite element mesh used in this simulation composes of 9,052 triangular elements with 44,015 degrees of freedom. 


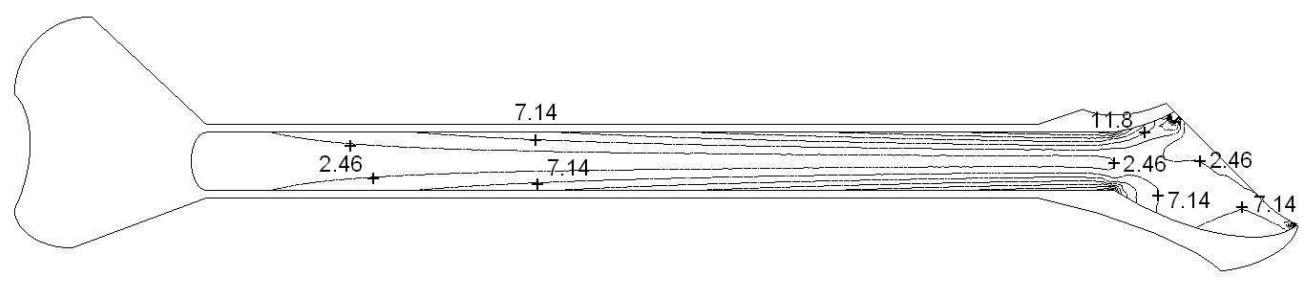

Figure 4: Contour plot of shear rate in the femoral canal.

The velocity of the PMMA flow at time $t=1 \mathrm{~s}$ is as shown in Figure 3. The velocity increases to the maximum value at the neck of the femoral canal and then continuously decreases while the cement goes along the femoral canal. The maximum and minimum velocities of the cement in the femoral canal are 8.810652 and $0.041458 \mathrm{~cm} / \mathrm{s}$, respectively. The shear rate in the femoral canal as shown in Figure 4 indicates that high shear rate occurs near the cancellous bone.

The pressure, for the optimal cement penetration into the cleaned cancellous bone, is found to be between 35 and $50 \mathrm{kPa}$ [11]. We therefore investigated the effect of inlet pressure on the flow pattern of the cement. Three different values of the inlet pressure in this study are chosen to be 20,35 and $50 \mathrm{kPa}$. Figure 5 shows the pressure along the central femoral canal and cancellous bone for three different pressure values. When the flow reaches the front surface of the cancellous bone, the pressure suddenly drops. Comparing the results obtained from three different pressure injection with 20, 35 and $50 \mathrm{kPa}$, we found that the larger pressure injection leads to the higher pressure jump. This extra higher pressure jump is created to impel the flow into the cancellous bone.

The mean flow velocity displayed in Figure 6 shows that an increasing zone of ascending motion of the cement flow is present in the first $3 \mathrm{~cm}$ from the inlet and the flow is then linearly decelerating to $0.023,0.042$ and $0.061 \mathrm{~cm} / \mathrm{s}$ at the front surface of the cancellous bone for the pressure 


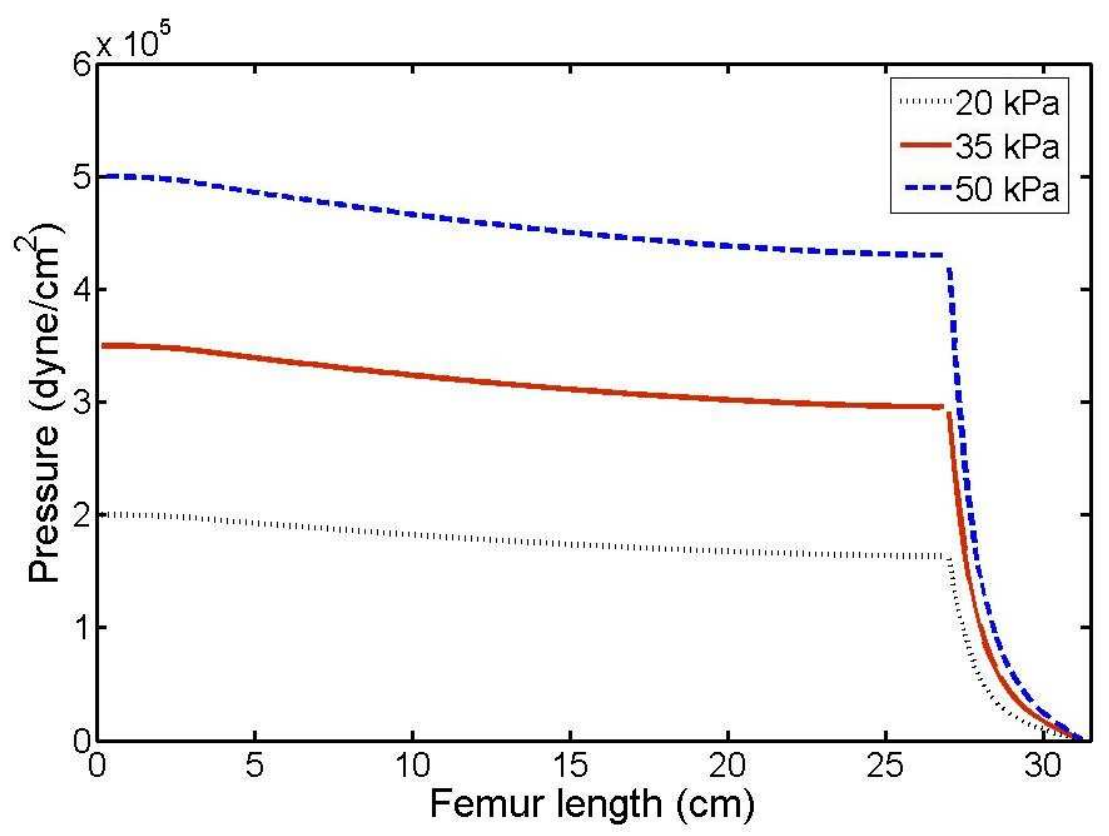

Figure 5: Pressure plot along the central femoral canal through cancellous bone where the dot line, the solid line and the dash line represent the results obtained from the model having the initial pressure of 20,35 and $50 \mathrm{kPa}$, respectively. 
injection of 20,35 , and $50 \mathrm{kPa}$, respectively.

\section{Conclusions}

A two dimensional numerical model for PMMA cement flow in the femoral canal and cancellous bone is developed. The model is used to study the effect of pressure injection on the flow in the femoral canal and the cancellous bone. The results appear to be in line with the experimental results that by increasing the pressure in the inlet, the velocity increases in the cancellous bone [5]. A rapid fall in pressure is created to impel the flow into the cancellous bone. The cement flow in the total hip replacement is an extremely complex phenomenon and there are many unsolved modelling problems such as the effect of heat transfer during the process. The present work only focuses on the cement flow in the femoral canal and the cancellous bone and ignores the effect of the heat release during the process. Further work should be carried out to study the three dimensional cement flow and heat transfer in the realistic domain.

Acknowledgments We are grateful to the Development and Promotion of Science and Technology Talented Project for the support of this research and also thank the Department of Orthopaedic Surgery, Faculty of Medicine Siriraj Hospital for providing some data for the analysis. We also thank the referees for their helpful comments and suggestions.

\section{References}

[1] P. H. Adegbile, B. Russery, L. Taylor, J. Tong. Failure of an uncemented acetabular prosthesis a case study, Engineering Failure 
(a)
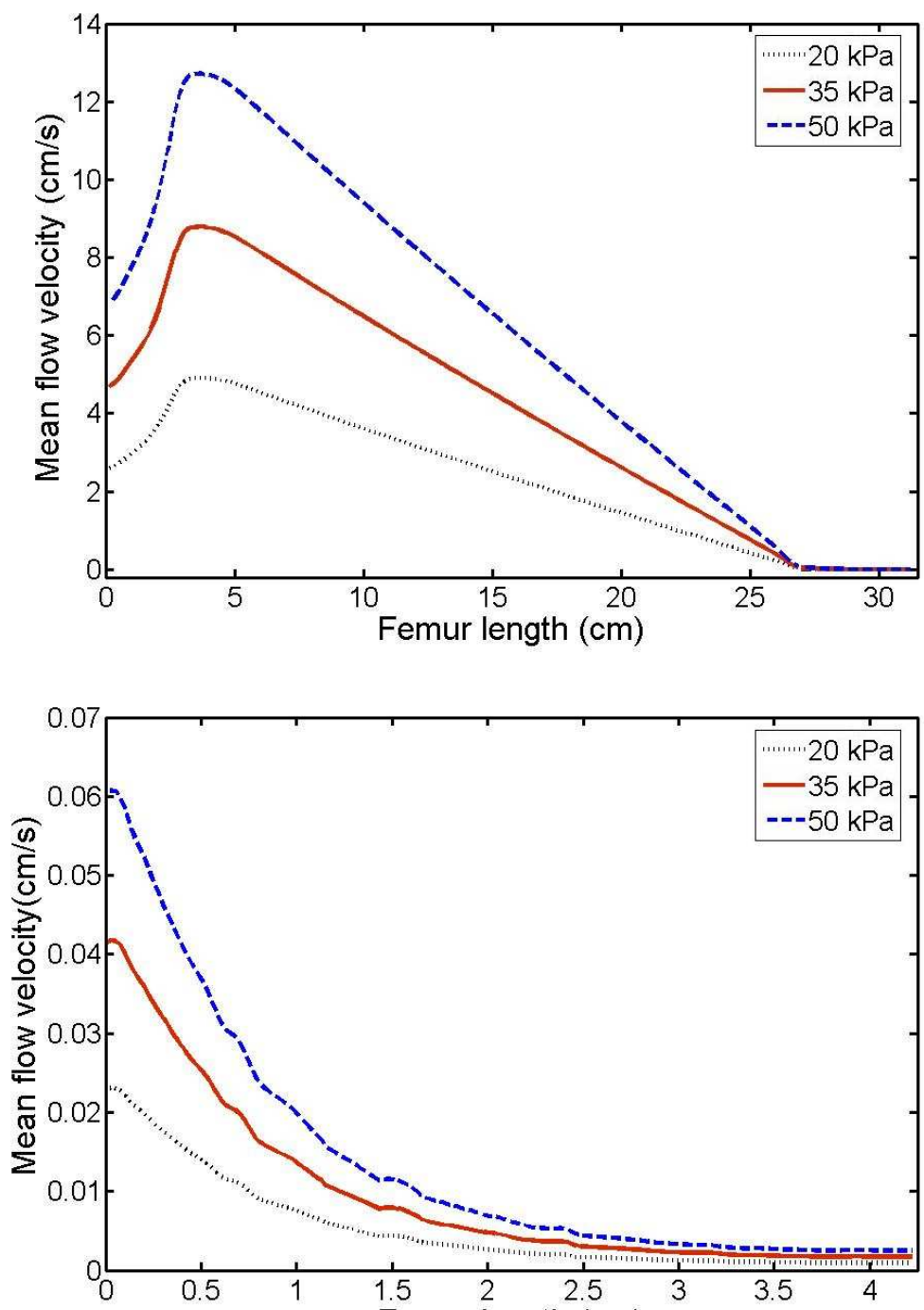

(b)

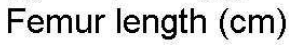

Figure 6: (a) velocity plot along the central femoral canal through the cancellous bone (b) velocity plot in the cancellous bone at distal epiphysis where the dot line, the solid line and the dash line represent the results obtained from the model having the initial pressure of 20,35 and $50 \mathrm{kPa}$, respectively. 
Analysis, 13(1), 2006, 163-169. C407

[2] G. Baroud, F. B. Yahia. A finite element rheological model for polymethylmethacrylate flow: analysis of the cement delivery in vertebroplasty, Prog. Instn Mech. Engrs Part H: J. Engineering in Medicine, 218, 2004. C407, C408

[3] Armand J. Beaudoin, William M. Mihalko, William R. Krause. Finite element modelling of polymethylmethacrylate flow through cancellous bone, J. Biomechanics 24(2), 1991, 127-136. C407, C408, C412

[4] S. L. Bevill, G. R. Bevill, J. R. Penmets, A. J. Petrella, P. J. Rullkoetter. Finite element simulation of early creep and ear in total hip arthroplasty, J Biomech, 38(12), 2005, 2365-2374. C407

[5] M. Bohner, B. Gasser, G. Baroud, P. Heini. Theoretical and experimental model to describe the injection of a polymethymethacrylate cement into a porous structure, Biometerials 24, 2003, 2721-2730. C407, C415

[6] Hanspeter Frei, Mohamed S. Gadala, Bassam A. Masri, Clive P. Duncan, Thomas R. Oxland. Cement flow during impaction allografting: a finite element analysis, Journal of Biomechanics, 39(3), 2006, 493-502. C407, C408, C412

[7] Michael J. Funk, Alan S. Litsky. Effect of cement modulus on the shear properties of the bone-cement interface, Biomaterials 19, 1998, 1561-1567. C407

[8] Do-Gyoon Kim, Mark A. Miller, Kenneth A. Mann. A fatigue damage model for the cement-bone interface, Journal of Biomechanics, 37, 2004, 1505-1512. C407

[9] A. B. Lennon, P. J. Prendergast. Residual stress due to curing can initiate damage in porous bone cement: experimental and theoretical evidence. Journal of Biomehanics 35(3), 2002, 311-321. C407 
[10] A. W. McCaskie, M. R. Barnes, E. Lin, W. M. Harper, P. J. Gregg. Cement pressurisation during hip replacement, Journal of Bone and Joint Surgery, 79(3), 1997, 379-384. C407, C408

[11] A. M. R. New, K. E. Tanner. In vivo measurement of acetabular cement pressurization using a simple new design of cement pressurizer, The Journal of Arthorplasty, 17(7), 1999. C412, C413

[12] B. Peter, N. Ramaniraka, L. R. Pakotomanana, P. Y. Zambelli, D. P. Pioletti. Peri-implant bone remodeling after total hip replacement combind with systemic alendronate treatment: a finite element analysis, Computer Methods in Biomechanics and Biomedical Engineering, 7(2), 2004, 73-78. C406

[13] M. A. Pérez, J. M. García, M. Doblaré. Analysis of the debonding of the stem-cement interface in intramedullary fixation using a non-linear fracture mechanics approach, Engineering Fracture Mechanics, 72, 2005, 1125-1147. C407

[14] M. Stańczyk. Study on modelling of PMMA bone cement polymerisation. Journal of Biomechanics, 38, 2005, 1397-1403. C407, C412

[15] A. W. L. Turner, R. M. Gillies, R. Sekel, P. Morris, W. Bruce, W. R. Walsh. Computational bone remodelling simulations and comparisons with DEXA Results, Journal of Orthopaedic Research 23, 2005, 705-712. C406 